\title{
Studies on Flat Sandwich-type Self-Powered Detectors for Flux Measurements in ITER Test Blanket Modules
}

\author{
Prasoon Raj, Maurizio Angelone, Toralf Döring, Klaus Eberhardt, Ulrich Fischer, Axel Klix, \\ Ronald Schwengner
}

\begin{abstract}
Neutron and gamma flux measurements in designated positions in the test blanket modules (TBM) of ITER will be important tasks during ITER's campaigns. As part of the ongoing task on development of nuclear instrumentation for application in European ITER TBMs, experimental investigations on self-powered detectors (SPD) are undertaken. This paper reports the findings of neutron and photon irradiation tests performed with a test SPD in flat sandwich-like geometry. Whereas both neutrons and gammas can be detected with appropriate optimization of geometries, materials and sizes of the components, the present sandwich-like design is more sensitive to gammas than $14 \mathrm{MeV}$ neutrons. Range of SPD current signals achievable under TBM conditions are predicted based on the SPD sensitivities measured in this work.
\end{abstract}

\section{INTRODUCTION}

$T_{p}$ HE breeding blanket will be a critical component of a power reactor based on nuclear fusion. Breeding of tritium for the fuel cycle of reactor, heat extraction and radiation shielding will be its main functions. To test the performance of such a blanket under representative conditions, test blanket modules (TBM) based on different blanket concepts will be inserted in designated equatorial ports of ITER reactor. As part of the nuclear experiments in TBMs during ITER campaigns, responses like tritium production, nuclear heating, material activation etc. will be measured at different locations in TBMs. The results of these experiments will be compared with those obtained from calculations. ITER being a large tokamak device with physical characteristics like those of a fusion power reactor, these tests will be used for a validation of the computational tools (e.g. Monte-Carlo and activation/inventory codes) and nuclear data employed in neutronic design studies of fusion reactor components.

Manuscript received June 1, 2017. The work leading to this publication has been funded partially by Fusion for Energy under the Specific Grant Agreement F4E-FPA-395-1. This publication reflects the views only of the authors, and Fusion for Energy cannot be held responsible for any use which may be made of the information contained therein.

Authors would like to gratefully acknowledge the support of scientists and staff at the laboratories in TRIGA reactor Mainz and ELBE accelerator of Helmholtz-Zentrum Dresden-Rossendorf (HZDR).

P. Raj, U. Fischer and A. Klix are with the Karlsruhe Institute of Technology (KIT), Hermann-von-Helmholtz-Platz 1, 76344 EggensteinLeopoldshafen, Germany (corresponding author e-mail: prasoon.raj@kit.edu).

M. Angelone is with the E.N.E.A. C.R., I-00044 Frascati (Rome), Italy.

T. Döring is with the Neutron Laboratory of Technical University of Dresden (TUD), 01328 Dresden, Germany.

$\mathrm{K}$. Eberhardt is with the Institute for Nuclear Chemistry, Johannes Gutenberg-University (JGU), 015228 Mainz, Germany.

R. Schwengner is with the Institute for Radiation Physics, HelmholtzZentrum Dresden-Rossendorf (HZDR), 01328 Dresden, Germany.
Online measurements of neutron and gamma fluxes will be important tasks in these experiments, since the TBMs will not be accessible during ITER campaigns. European Union (EU) has two proposed TBM systems to be installed in ITER and tested: the HCLL and the HCPB TBMs [1]. With high radiation, high temperature, and electromagnetic interferences the operating conditions for nuclear detectors in TBM are detrimental. Development of suitable nuclear instrumentation for such harsh conditions is an ongoing project. Activation foils, diamond detectors and self-powered detectors (SPD) are among the detector classes chosen for further study.

Self-powered detectors are commonly utilized as flux monitors in fission power reactors. They have compact and robust design and they do not require external bias voltage, making them highly desirable for application in regions with less accessibility like nuclear reactor cores. They are often composed of standard metals, mineral oxides and alloys, making them desirable with respect to nuclear nonproliferation and export-control guidelines. For these reasons, some regular SPDs, one commercially manufactured ad-hoc SPD [2] and several different variants in flat sandwich-like design [3] are under investigation.

\section{Development of Test Detector}

\section{A. Description of a Self-Powered Detector}

An SPD is a multi-layered device [4] with two electrodes separated by a mineral oxide insulation layer. There is a central emitter layer with high neutron or gamma interaction cross-section. Under these interactions, the material produces high energy electrons. These electrons move outwards and are stopped in the outer electrode layer called collector. The separation of charges forms the detector signal. The response is usually measured as a direct current (DC) signal. The rate of neutron/gamma interactions, thereby the rate of electron emission and magnitude of DC signal are proportional to the incident flux. Sensitivity of an SPD is defined as SPD current per unit of incident flux, expressed in units of $\mathrm{A} \mathrm{cm}^{2} \mathrm{~s}^{1}$.

Traditionally, SPDs are made in a coaxial cylindrical geometry. A wire (or rod) of emitter is packed in a closed tube of collector with a tube or beads of mineral oxide between them for electrical insulation. It can also be designed in a flat geometry with foils of insulator and collector sandwiching a foil of emitter. A schematic of this design is shown in Fig. 1 with the current measurement connections.

The variation of SPD signal under dynamic reactor conditions has a profile in time. This is based on the half-life corresponding to the exact atomic or nuclear process which creates most of the fast electrons. The most commonly used 
SPDs in fission reactor cores, known as Delayed Self-Powered Neutron Detector (SPND) respond mainly via (n, $\left.\beta^{-}\right)$process. In this process, neutron absorption reaction leads to formation of a radioactive daughter nuclide in the emitter that undergoes beta minus decay. Due to the half-life of beta decay, the signal does not vary promptly with variation in incident flux. The alternative (n, $\left.\gamma, e^{-}\right)$process involves emission of high-energy gamma in the neutron-activated emitter material and subsequent interactions through photoelectric effect, Compton scattering and pair-production routes to produce fast secondary electrons, which form the detector signal. This is a prompt process, making the so-called Prompt SPND a good choice for reactor power-level monitoring. A third common process, $\left(\gamma, \mathrm{e}^{-}\right)$process is the principle at the heart of a SelfPowered Gamma Detector (SPGD). In this, external gammas from the surrounding of the detector are detected through secondary electron emission in the emitter. Usually, a detector is optimized for high response to measure neutrons or gammas. Nevertheless, the radiation field in which an SPD is applied is mixed with both neutrons and gammas and the output signals are mixed with contributions from both.

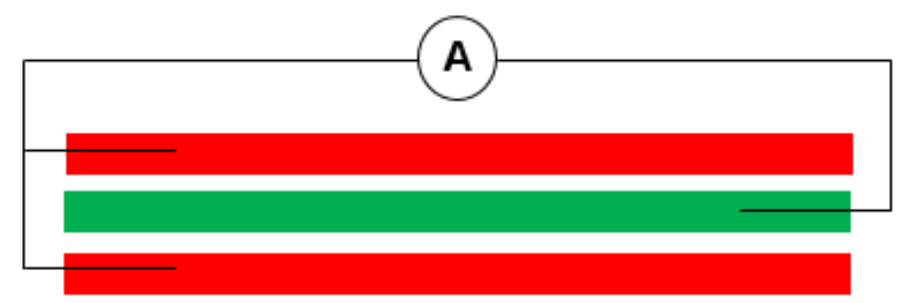

Fig. 1. Schematic of an SPD showing emitter layer in green, insulation in white and collector layers in red. An ammeter across emitter and collector (grounded) can be used to see the DC signal from the detector.

\section{B. Design and Construction of Flat Sandwich-like SPD}

Test blanket modules (TBM) of ITER have neutrons and gammas over a wide energy range [1]. The neutrons range from thermal energy to $14 \mathrm{MeV} \mathrm{D}-\mathrm{T}$ neutron energy. Majority of them have intermediate energies. Total neutron flux intensity can go up to the order of $10^{14} \mathrm{n} \mathrm{cm}^{-2} \mathrm{~s}^{-1}$. Unfortunately, no available neutron sources provide comparable field characteristics for detector tests. Gammas at positions relevant to SPDs range in energies between few $\mathrm{keV}$ to $30 \mathrm{MeV}$, intensities going up to the order of $10^{13} \gamma \mathrm{cm}^{-2} \mathrm{~s}^{-1}$. Dedicated detectors for measurement of TBM neutrons (SPND) and gammas (SPGD) are vital. To this end, theoretical and experimental investigations are underway in European laboratories [2, 3].

Based on a set of criteria, several materials were chosen for emitter of a delayed SPND for TBM [2]. These criteria included high saturation activity, short half-life of product nuclide and high beta energy. Designing a pure delayed SPND for TBM neutrons is challenging because of the relatively lower reaction cross-sections towards fast neutrons. On the other hand though, the probability of photon production is higher than that of beta emission for fast neutrons. So, prompt current due to neutron-induced gammas in detector dominates the signal. This is unlike the case of thermal neutrons, where both the processes have similar cross-sections, and the delayed current dominates for short half-life beta decays. Overall sensitivity of SPND for fast neutrons is lower. Another important difference is that possibility of threshold reactions gives a way to exclusively measure fast neutron contribution in TBM. Beryllium, vanadium, chromium and a few other materials are chosen for investigation as emitter of SPDs for TBM. For insulation alumina and for collector Inconel-600 alloy are used in these studies.

A test detector (Fig. 2) in flat geometry was designed with foils of collector and insulator sandwiching a foil of emitter. High-purity foils with dimensions $25 \mathrm{~mm}$ by $25 \mathrm{~mm}$ by 0.5 to $2 \mathrm{~mm}$ were used in different experiments. The model is flexible with openable parts to test different material combinations in different thicknesses. The assembled detector is packaged in a case of aluminum which also works as an electromagnetic shield. SPDs in flat geometry are of interest for application in TBMs. A foil of emitter can be encapsulated in a case made from collector material with insulation material filling the gap. One can think of a multi-layer strip-like detector or a chip-like compact detector which can be integrated with minimal invasion.

Although multiple materials have been tested and studied with this detector, results recorded only with vanadium SPD are presented in the upcoming section. Vanadium is a good reference material to study the newly implemented flat geometry, as its response is simple and well-understood for thermal neutrons. It is a candidate emitter for SPNDs [2] in TBMs, expected to provide information on thermal as well as fast neutrons. Also, it is a relatively easy material to handle in the laboratory than beryllium and chromium. The detector (VSPD) has $1 \mathrm{~mm}$ thick layers of vanadium emitter and Inconel600 collector, and $0.5 \mathrm{~mm}$ thick alumina insulator.

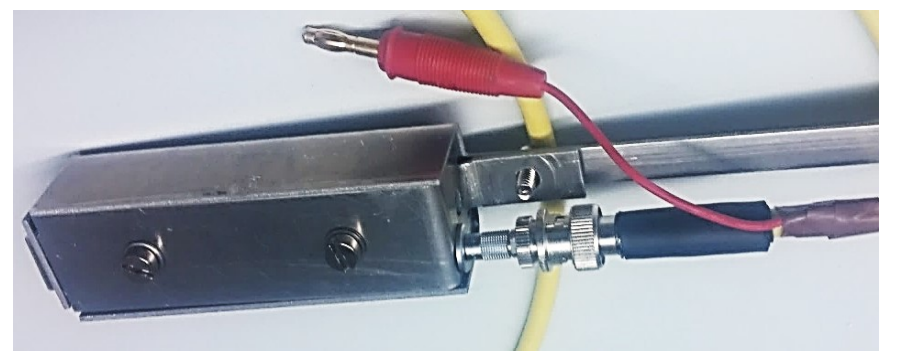

Fig. 2. Constructed flat sandwich-type SPD with an electromagnetic case made from aluminum and coaxial cable connection for signal

\section{IRRADIATION TESTS AND RESUltS}

The V-SPD has been irradiated with three test sources: thermal neutrons, $14 \mathrm{MeV}$ fast neutrons and bremsstrahlung photons. Small DC signals (50 fA to $500 \mathrm{pA}$ ) have been measured in these tests. The flat SPD currents are proportional to the incident neutron [3] and gamma flux, as required for a flux monitor. It has been established in tests with multiple material combinations and flux levels varying over orders of magnitude. At small currents, there are chances of disturbances due to various currents produced in mechanical, electromagnetic, thermal and radiation induced effects in detector and cables. The measurements have been performed with care, reducing these sources as far as possible. 
A proof of the working of flat sandwich-type SPD is achieved by irradiating the V-SPD in the TRIGA Mark II reactor of the Johannes-Gutenberg University Mainz. Experimental position is near the hot end of the graphite thermal column (GTC) of the reactor with total thermal neutron flux of the order of $10^{10} \mathrm{n} \mathrm{cm}^{-2} \mathrm{~s}^{-1}$ [5]. The building up and saturation of the signal during first few minutes of irradiation and its decay after shutdown of the reactor showed characteristics of the vanadium emitter $\left(\mathrm{T}_{1 / 2}=3.75 \mathrm{~min}\right)$ like expected. Presence of a prominent prompt component in the signal shows enhanced sensitivity to gammas produced in the reactor and detector materials. Sensitivity of V-SPD calculated at saturation (Table I) is of the same order as the commonly reported sensitivities of cylindrical SPNDs in thermal reactor.

\section{Table I}

Measured saturated currents, incident particle flux intensities (measured with $\mathrm{Au}$ foil activation in thermal neutron field, with calibrated $\alpha$-detector in fast neutron field and estimated with validated Monte-Carlo simulation in bremsstrahlung photon field) and sensitivities of V-SPD in three test scenarios

\begin{tabular}{l|c|c|c}
\multicolumn{1}{c|}{ Test Field } & $\begin{array}{c}\text { Current } \\
\mathbf{1 0}^{-12} \mathbf{A}\end{array}$ & $\begin{array}{c}\text { Flux } \\
\mathbf{1 0} \mathbf{c m}^{-\mathbf{2}} \mathbf{s}^{-\mathbf{1}}\end{array}$ & $\begin{array}{c}\text { Sensitivity } \\
\mathbf{1 0}^{-\mathbf{2 0}} \mathbf{A} \mathbf{~ c m}^{\mathbf{2}} \mathbf{~}\end{array}$ \\
\hline Thermal neutrons & 492 & 26.8 & 1.84 \\
\hline Fast Neutrons & 6.48 & 1.56 & 0.42 \\
\hline Photons & 6.20 & 0.06 & 9.60
\end{tabular}

For fast neutron irradiation, $14 \mathrm{MeV}$ Neutron Generator of Technical University of Dresden (TUD-NG) is utilized. TUD$\mathrm{NG}$ is an accelerator based intense D-T neutron source situated in Dresden-Rossendorf. It provides a neutron spectrum with peak around $14 \mathrm{MeV}$ and flux intensity going up to $10^{10} \mathrm{n} \mathrm{cm}^{-2} \mathrm{~s}^{-1}$ near the tritium target. In fast neutron field, the detector signal rises and disappears almost instantaneously with the neutron flux. The delayed current component is negligibly small (approx. $6.3 \%$ of the total). It is a sum of contributions from ${ }^{51} \mathrm{Ti}$ and ${ }^{52} \mathrm{~V}$ beta electrons, betas from aluminum case, collector and insulator materials and decay gamma from activated detector and surrounding materials.

The TUD-NG is characterized by a mixed neutron-gamma field. An estimation has been made in a Monte-Carlo model calculation of the generator, for the ratio of intensities of neutrons and gammas. At the experimental position, total gamma flux intensity is approx. $19 \%$ of the total neutron flux intensity. To obtain the neutron to gamma signal ratio for the tests at TUD-NG, a comparable test in a pure gamma field is required. Whereas radioisotope based line gamma sources are frequently used for such tests, a photon source is used in this work. At the beam dump of the ELBE [6] accelerator of Helmholtz-Zentrum Dresden-Rossendorf, there is a high intensity high-energy bremsstrahlung photon source created which is used for photo-activation experiments. The ELBE photon source has a continuous energy spectrum ending close to the energy of the electron beam. Beam parameters are selected ( $I_{\text {beam }}=0.5 \mu \mathrm{A}, \mathrm{E}_{\text {beam }}=10 \mathrm{MeV}$ ) to have an energy spectrum of photons close to the one of gammas in TUD-NG. SPD current is proportional to the photon flux, showing again that the detector has linear and high response to photons.

The pure gamma sensitivity measured in ELBE irradiation of V-SPD (Table I) is substantially higher than the optimistic thermal neutron sensitivity assessed in case of TRIGA irradiation. On multiplying this with the calculated total flux of gammas (having energy spectrum similar to ELBE beam dump) at the experimental position in TUD-NG, one obtains that about $81 \%$ of the signal in TUD-NG is due to detection of gamma. With the rest $19 \%$ of the signal, the $14 \mathrm{MeV}$ neutron sensitivity of V-SPD is determined to be approx. $4.15 \times 10^{-21}$ $\mathrm{A} \mathrm{cm}^{2} \mathrm{~s}^{-1}$ as reported in Table I. Therefore, in $14 \mathrm{MeV}$ neutron field, the sandwich-type V-SPD has a higher sensitivity towards gammas than neutrons.

It is clear that flat SPDs can be applied as SPGD for gamma measurements in TBM. Signals in the range of nA to $\mu \mathrm{A}$ can be expected with the present design. This can be increased by changing emitter material to a high-Z metal, and further optimizing the geometry. On the other hand, the neutron detection is also feasible but it is tricky. Thermal neutron fluxes can be measured by separating the delayed currents due to thermal capture reaction(s). But for fast neutrons, an indirect mechanism can be used in which neutron-induced gammas are detected in a prompt SPND. Essential design improvements and further tests are required before optimized prototype SPDs for application in TBM can be realized.

\section{CONCLUSions}

As part of the ongoing task on development of nuclear instrumentation for European test blanket modules of ITER, self-powered detectors in flat geometry have been constructed. The present sandwich-like vanadium SPD in aluminium casing is responsive to neutrons and gammas. The currents expected with this detector in TBM environment can be reliably measured. Gammas can be measured directly with such a detector, while fast neutrons need to be measured indirectly through neutron-induced prompt gammas.

\section{REFERENCES}

[1] P. Calderoni, "Status of the HCLL and HCPB Test Blanket System instrumentation development," 21st Top. Meet. Technol. Fusion Energy (TOFE), 9-13 Novemb. 2014, Anaheim, CA.

[2] M. Angelone, A. Klix, M. Pillon, P. Batistoni, U. Fischer, and A. Santagata, "Development of self-powered neutron detectors for neutron flux monitoring in HCLL and HCPB ITER-TBM," Fusion Eng. Des., vol. 89, no. 9-10, pp. 2194 2198, 2014.

[3] P. Raj, M. Angelone, U. Fischer, and A. Klix, "Self Powered Detectors for Test Blanket Modules in ITER," in IEEE Nuclear Science Symposium, 29 Oct. - 5 Nov. 2016, Strassbourg, France, 2016.

[4] J. W. Hilborn, "Self-Powered Neutron Detectors for Reactor Flux Monitoring," Nucleonics, vol. 22, pp. 69-74, 1964.

[5] B. Wortmann, "Ph.D. Thesis: Auslegung und Optimierung einer Bestrahlungseinrichtung fuer die Bor-

Neutroneneinfangtherapie an autotransplantierten Organen," Technical Unversity of Dresden, 2008.

[6] R. Schwengner, R. Beyer, F. Dönau, E. Grosse, A. Hartmann, A. R. Junghans, S. Mallion, G. Rusev, K. D. Schilling, W. Schulze, and A. Wagner, "The photonscattering facility at the superconducting electron accelerator ELBE," Nucl. Instruments Methods Phys. Res. Sect. A Accel. Spectrometers, Detect. Assoc. Equip., vol. 555, no. 1-2, pp. 211-219, 2005. 